\title{
Modélisation des sources de bruit dans les dispositifs MOS
}

\section{A. Guen Bouazza*, H. Sahraoui, B. Bouazza, K. E. Ghaffour et N. E. Chabane Sari}

Unité de recherche des matériaux et énergies renouvelables, faculté des sciences de l'ingénieur, Département d'Electronique, Université Abou-Bekr-Belkaid de Tlemcen, BP 119, Tlemcen 13000, Algérie.

(Reçu le 03 Juin 2005, accepté le 30 Août 2005)

* Correspondance et tirés à part, e-mail : guenahlam@yahoo.fr

\section{Résumé}

Nous présentons dans ce travail les origines physiques des deux sources principales du bruit dans le transistor MOS (métal-oxyde-semiconducteur), et leur modélisation. Dans la première partie, nous nous intéressons au bruit en $l / f$ dit le "flicker noise". La seconde partie de ce travail porte sur le bruit thermique et l'impact de la longueur du canal sur ce bruit. Le bruit induit au niveau de la grille dit "induced gate noise" est également présenté.

Mots-clés : Bruit en I/f, bruit thermique, modélisation, modulation, longueur du canal, bruit induit, grille, MOS, MOSFET.

\section{Abstract}

\section{Modeling of noise sources in MOS devices}

We present in this work an investigation of the physical origins of the two main noise sources in MOS devices. In the first part, we interest to the flicker noise and its detailed derivation's. The thermal noise is discussed in the second part with the explanation of the channel length impact in this noise. At next, we present briefly the induced gate noise.

Keywords : Flicker noise, thermal noise, modeling, modulation, channel length, induced noise, gate, MOS, MOSFET. 


\section{1 - Introduction}

La maîtrise de la technologie de fabrication des transistors CMOS (complementary metaloxyde-semiconductor), leur miniaturisation en relation avec un coût réduit incitent de plus en plus les spécialistes à entrevoir des applications en radiofréquence. Les applications modernes de cette technologie dans cette bande de fréquence imposent une modélisation correcte du bruit afin d'assurer un bon fonctionnement. Les deux sources majeures de bruit dans un transistor MOS sont le bruit en $l / f$ (basse fréquence) et le bruit thermique. En radiofréquence le bruit en $l / f$ est négligeable, le bruit thermique devient alors la principale source de bruit.

Nous présentons dans cet article les origines physiques de ces deux sources de bruit. Les théories expliquant le phénomène du bruit en $l / f$ font l'objet de la première partie de ce travail, la seconde partie est consacrée à la présentation du bruit thermique et les démonstrations de la densité spectrale illustrant le comportement de ce bruit. L'impact de la longueur du canal sur le bruit thermique fait l'objet de la partie suivante. Enfin nous présentons brièvement le bruit induit dans la grille.

\section{2 - Le bruit en $I / f$}

Dans la littérature on utilise le terme " bruit en $l / f$ " pour tous les bruits en basse fréquence. Dans cette gamme de fréquence ce bruit est prédominant, et combien même ce bruit est omniprésent, aucun mécanisme universel n'a été prouvé définitivement comme étant sa cause. En d'autres termes, ce bruit peut être modélisé empiriquement mais ne peut pas être a priori prévu. Ce bruit est sensiblement plus grand dans les transistors MOS en comparaison avec les bipolaires où il est principalement conditionné par l'état d'interface à la jonction constituée par la couche d'oxyde de grille et le canal de conduction. L'origine du bruit en $l / f$ est expliquée par les théories suivantes:

\section{2-1. Fluctuations du nombre de porteurs de charge}

Cette théorie a été proposée par Mc Worther [1]. Ce bruit est dô au piégeage des porteurs de charge au-dessous du diélectrique au niveau de la grille. Suite à ses travaux effectués au laboratoire Lincoln, il cita :

"Les résultats actuels ne laissent maintenant aucun doute que ce bruit est globalement, sinon entièrement un phénomène de surface 11.

Ainsi, cette théorie explique la fluctuation du nombre de porteurs de charge $(\Delta \mathrm{n})$ par leur piégeage au niveau de l'interface oxyde-grille, d'où l'apparition d'un bruit dit "I bruit en $l / f$ II. Ce dernier est représenté analytiquement par la densité spectrale du courant qui circule au niveau du drain, notée $S_{10}$ et donnée par : 


$$
S_{I_{D}}=\frac{K_{F} q^{4} I_{D}^{2}}{n^{2} k T W L f} \frac{N_{S T}}{\left(C_{o x}+C_{S S}+C_{I}\right)^{2}}\left(\frac{\mu_{e f f}}{\mu_{0}}\right)^{2}
$$

avec:

$$
\mu_{e f f}=\frac{\mu_{0}}{1+\theta\left(V_{G S}-V_{T}\right)}
$$

Où :

$K_{F}:$ constante dépendant de la technologie utilisée

$n$ : concentration des électrons

$q$ : la charge de l'électron

$I_{D}:$ le courant du drain

$k$ : la constante de Boltzmann

$W$ : la largeur du canal

$L$ : la longueur du canal

$I$ : la température en degré Kelvin

$N_{S T}$ : la densité des porteurs piégés en surface

$C_{s s}$ : la capacité d'interface

$C_{1}:$ la capacité d'inversion

$C_{o x}$ : la capacité de l'oxyde

$V_{G S}$ : la tension entre la grille et la source

$V_{T}$ : la tension de seuil uthreshold "

$\mu_{\text {eff }}$ : la mobilité effective des porteurs de charge

$\mu_{0}$ : la mobilité des porteurs de charge à faible champ

$\theta$ : le facteur d'atténuation de la mobilité des porteurs de charges dans le canal.

\section{2-2. Fluctuation de la mobilité}

Hooge [l] attribue le bruit en $l / f$ à la fluctuation de la mobilité des porteurs de charge. II développa une formule empirique déterminant le bruit en $l / f$ en fonction de la variation de la résistance du canal. La densité spectrale du bruit est alors donnée par la relation suivante :

$$
R_{n}^{2}=\frac{\alpha_{H}}{N} \frac{R^{2}}{f}
$$

avec Nle nombre de porteurs de charges libres au niveau du "I bulk I, et $\alpha_{\mathrm{H}}$ la constante de Hooge $\left(\alpha_{\mathrm{H}}=2.10^{-3}\right)$. En se basant sur ces résultats, il conclut [1]:

"I Les recherches effectuées démontrent que le bruit en l/f est dû à la fluctuation de la mobilité des porteurs de charge et non pas à la variation de leur concentration II. 


\section{2-3. Modèle unifié}

La fluctuation de la mobilité et la variation du nombre de porteurs de charge ont la même origine d'où leur complète corrélation [1]. Cette théorie étend l'hypothèse de la variation du nombre de porteurs de charge à la distribution des charges libres au niveau de l'oxyde. Par conséquent, non seulement le nombre de porteurs de charges varie mais leur mobilité varie aussi. Un tel modèle décrit avec précision le mécanisme du bruit dans les transistors NMOS et PMOS. Plusieurs modèles tels que le MOS modèle 9, le MOS modèle $I l$ ainsi que le $B S I M 3 v 2$ et $B S I M 4$ utilisent le modèle unifié [1].

Afin de mettre en évidence le bruit basse fréquence, nous avons élaboré un programme qui permet de calculer la densité spectrale du bruit; notre choix s'est porté sur les transistors MOS de dernière génération, soit le $M M I l$, modèle développé par Philips. C'est un modèle compact développé pour des applications RF. II a été spécialement développé pour donner une meilleure description des courants et des charges ainsi que leurs premières dérivées (transconductances, conductance et capacité), et le BSIM4, modèle de transistors développés par l'Université de Californie de Berkeley (UCB). BSIM4 est le successeur du BSIM3 par rapport auquel il présente les améliorations suivantes:

- nouveau modèle intrinsèque de la résistance d'entrée.

- nouveau modèle du bruit thermique.

- modèle non quasi-statique NQS.

Les résultats de simulation obtenus pour ces deux modèles sont illustrés à la Figure 1.
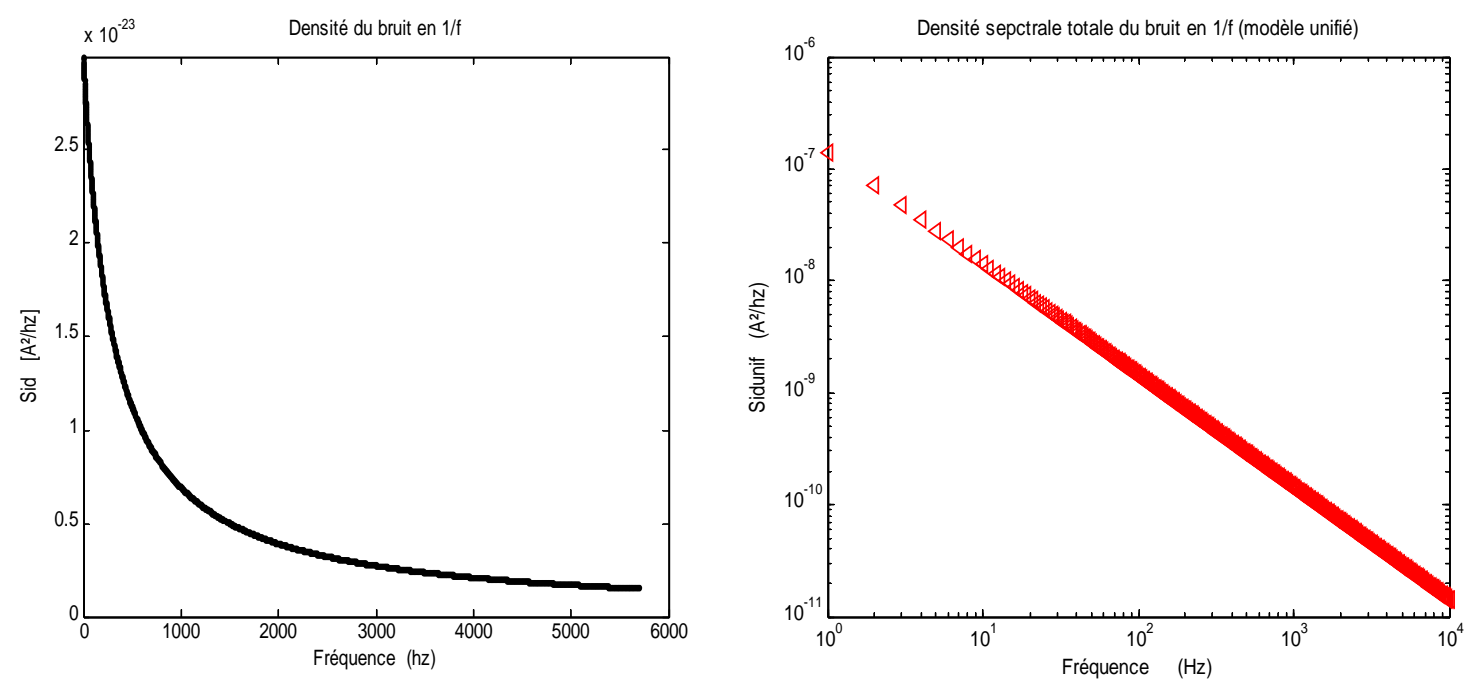

(a)

Figure 1 : Densité spectrale du bruit pour le modèle MMII,

(b)

(a) échelle linéaire, (b) échelle logarithmique 
A la Figure 1, on représente la densité spectrale du bruit pour un transistor MOS de type $M M I l$, en échelle linéaire (Figure $\mathbf{l a}$ ) et en échelle logarithmique (Figure $\mathbf{l b}$ ). L'exploitation de ces courbes nous permet d'affirmer la présence du bruit en $l / f$ car la densité spectrale est bien inversement proportionnelle à la fréquence, et ceci tant que la fréquence reste inférieure à une valeur précise appelée $f_{c}$ (f corner) qui dans le cas de notre modèle est de l'ordre de $1 \mathrm{KHz}$. On peut aussi remarquer que ce transistor présente un faible niveau de bruit (peu bruyant), ce qui était prévisible du fait qu'il a été conçu dans ce sens.

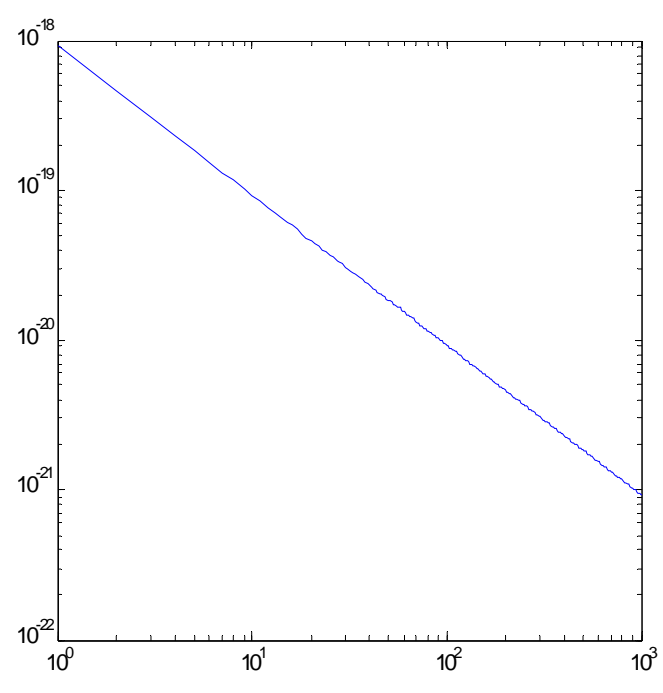

Fréquence (Hz)

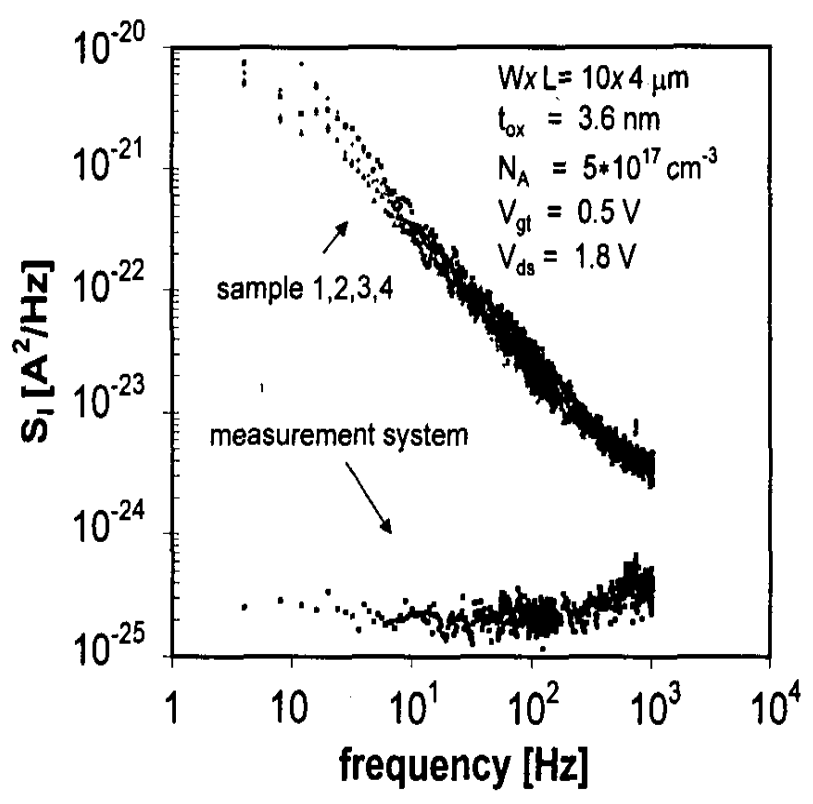

(b)

(a)

Figure 2 : Densité spectrale du bruit pour le modèle BSIM4, (a) résultats de simulation, (b) résultats expérimentaux [ 8].

A la Figure 2, on représente la densité spectrale du bruit pour un transistor MOS de type BSIM4 en échelle logarithmique (Figure 2a). L'exploitation de ces courbes nous permet d'affirmer la présence du bruit en $l / f$ car la densité spectrale est bien inversement proportionnelle à la fréquence, et ceci tant que la fréquence reste inférieure à une fréquence de $1 \mathrm{KHz}$.Ce transistor est aussi peu bruyant. Une étude expérimentale a permis de représenter la densité spectrale du bruit [8]. Les résultats de simulation que nous avons obtenu pour ce modèle sont satisfaisants en comparaison avec les résultats expérimentaux de la Figure $\mathbf{2 b}$ [ 8]. 


\section{Expression de la densité spectrale du bruit en $I / f$ pour le modèle $M M I I$}

Pour étudier la densité spectrale du transistor MMIl, nous présentons les différentes étapes de calcul donnant la relation générale du bruit en $\mathrm{l} / \mathrm{f}$. Ce modèle utilise le potentiel de surface $\Psi_{\mathrm{S}}$ dans la formulation de ces équations, ce qui amène à des expressions valides dans tous les régimes de fonctionnement. La densité spectrale du bruit au niveau du drain du transistor est exprimée par [6] :

$$
S_{I_{D}}=\frac{K_{B} T I_{D S}^{2}}{\gamma_{o x} f W L^{2}} \int_{0}^{L} n_{t}\left(E_{f n}\right)\left[\frac{R}{N(x)} \pm \alpha_{S} \mu_{e f f}\right]^{2} d x
$$

où $n_{t}\left(E_{f n}\right)$ représente une distribution approximative de la densité des pièges dans l'oxyde en fonction de l'énergie, $\alpha_{S}$ est le coefficient de répartition de la mobilité, $\gamma_{o x}$ est un coefficient d'atténuation, $K_{B}$ la constante de Boltzmann, $T$ est la température, IDs est l'intensité du courant circulant de la source vers le drain, $W$ et $L$ représentent respectivement la largeur et la longueur du canal, $N$ est le nombre de porteurs de charges dans le canal par unité de surface, donné par $N=-Q_{i n v} / q ; R$ représente le rapport entre la variation du nombre de porteurs de charges et la variation du nombre de pièges occupés, il est exprimé par :

$$
R \equiv \frac{\partial N}{\partial N_{t}}
$$

En régime de forte inversion $\mathrm{R}=-1$ et en régime de faible inversion $\mid \mathrm{R} K<1$. En régime de faible inversion on peut écrire :

$$
\partial Q_{g}+\partial Q_{b}+\partial Q_{i n v}+\partial Q_{t}=0
$$

0ù

$$
\mathrm{R} \equiv \frac{\partial \mathrm{N}}{\partial \mathrm{N}_{\mathrm{t}}} \simeq \frac{\mathrm{Q}_{\text {inv }} / \Phi_{\mathrm{T}}}{\mathrm{C}_{\mathrm{g}}+\mathrm{C}_{\mathrm{b}}-\frac{\mathrm{Q}_{\text {inv }}}{\Phi_{\mathrm{T}}}}
$$

Où :

Qg : la densité de charge dans la grille,

$Q_{b}$ : la densité de charge dans le bulK,

Qt : la densité de charge totale,

Qinv : la densité de charge d'inversion qui varie en fonction de la tension appliquée à la grille.

En faible inversion, $\frac{Q_{i n v}}{\partial \Psi_{S}} \approx-\frac{Q_{i n v}}{\Phi_{T}}$ où $\Phi_{\mathrm{T}}$ est le potentiel thermique, $R$ peut enfin être exprimé par : 


$$
R=-\frac{N}{N+N^{*}}
$$

avec

$$
N^{*}=\frac{\Phi_{T}}{q}\left[C_{g}+C_{b}\right] \approx \frac{\Phi_{T}}{q} C_{i n v}
$$

Afin de résoudre la densité spectrale $S_{10}$ nous devons expliciter la relation entre $\alpha_{S}, \mu_{\text {eff }}$ et $n_{t}\left(E_{f n}\right)$. Adoptons alors l'écriture suivante :

$$
\begin{aligned}
n_{t}^{*}\left(E_{f n}\right) & =n_{t}\left(E_{f n}\right)\left[1 \pm \alpha_{s} \mu_{e f f} \frac{N}{R}\right]^{2}=A+B N+C N^{2} \\
A & =n_{t}\left(E_{f n}\right), B= \pm \frac{2 \alpha_{S}}{R} n_{t}\left(E_{f n}\right) \mu_{e f f}, C=n_{t}\left(E_{f n}\right)\left(\frac{\alpha_{s} \mu_{e f f}}{R}\right)^{2}
\end{aligned}
$$

Plus encore, $d V$ peut être écrit en fonction de $d N[6]$ :

$$
\begin{aligned}
& d V=\frac{q}{C_{i n v}} \frac{\partial V}{\partial \Psi_{S}} d N \\
& \frac{\partial V}{\partial \Psi_{S}}=1-\frac{\Phi_{T}}{Q_{i n v}} \frac{\partial Q_{i n v}}{\partial \Psi_{S}}=\frac{N+N^{*}}{N}
\end{aligned}
$$

Ainsi nous pouvons écrire l'expression (4) sous la forme suivante :

$$
S_{I_{D}}=\frac{K_{B} T I_{D S}^{2}}{\gamma_{o x} f W L^{2}} \int_{V_{S B}}^{V_{D B}} n_{t}\left(E_{f n}\right)\left[1 \pm \alpha_{S} \mu_{\text {eff }} \frac{N}{R}\right]^{2} \frac{R^{2}}{N} d V
$$

En régime de saturation la modulation du canal peut influencer la densité spectrale. En tenant compte de ce nouveau paramètre la densité spectrale s'exprime par :

$$
S_{I_{D}}=\frac{K_{B} T I_{D S}^{2}}{\gamma_{o x} f W L^{2}} \int_{0}^{L-\Delta L} n_{t}^{*}\left(E_{f n}\right) \frac{R^{2}}{N^{2}} d x+\int_{L-\Delta L}^{L} n_{t}^{*}\left(E_{f n}\right) \frac{R^{2}}{N^{2}} d x
$$

Nous avons cité au début de ce paragraphe que le modèle de bruit que nous avons adopté est valable pour tous les régimes de fonctionnement. Les résultats obtenus par le programme de simulation que nous avons élaboré permettent de confirmer cette hypothèse. Ces résultats sont représentés par le graphe de la Figure 3.

A la Figure 3, on représente la densité spectrale du bruit pour un transistor MOS de type $M M I l$ pour un modèle unifié dans ses différentes régions de fonctionnement où le bruit basse fréquence apparaît pour les fréquences basses $f<f_{c}$, et le bruit thermique pour $f>f_{c}$. L'analyse des courbes obtenus permet de constater que le bruit thermique est plus important en région II subthreshold II, ce qui était prévisible. 

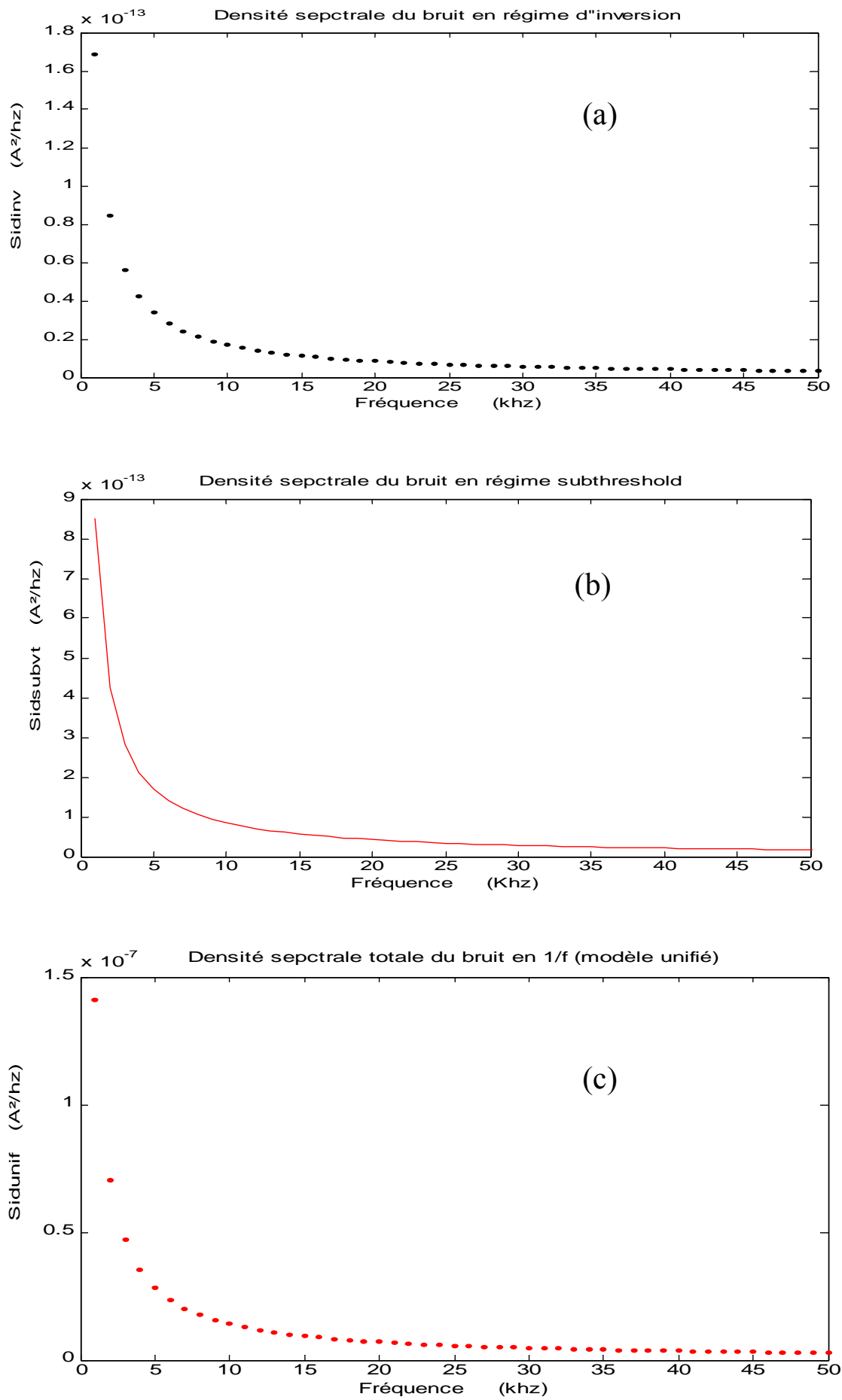

Figure 3 : Illustration du bruit en I/f pour les différentes régions

(a) région d'inversion, (b), région de subthreshold, (c) bruit total 


\section{Modulation de la longueur du canal}

Quand la tension $\mathrm{V}_{D S}$ augmente au-delà de $\mathrm{V}_{\text {DSsat }}$ le canal se rétrécit avec une distance $\Delta \mathrm{L}$ (Figure 4) et ceci est dô au déplacement du point de saturation de la vitesse des porteurs de charges dans le canal vers la source [6]. Le calcul de cette distance nécessite la résolution de l'équation de Poisson à deux dimensions donnée par :

$$
\operatorname{div} \mathrm{E}(\mathrm{x}, \mathrm{y})=\frac{\rho(\mathrm{x}, \mathrm{y})}{\varepsilon}
$$

Où $E$ est le champ électrique, $\rho$ la densité de charge, et $\varepsilon$ la permittivité.

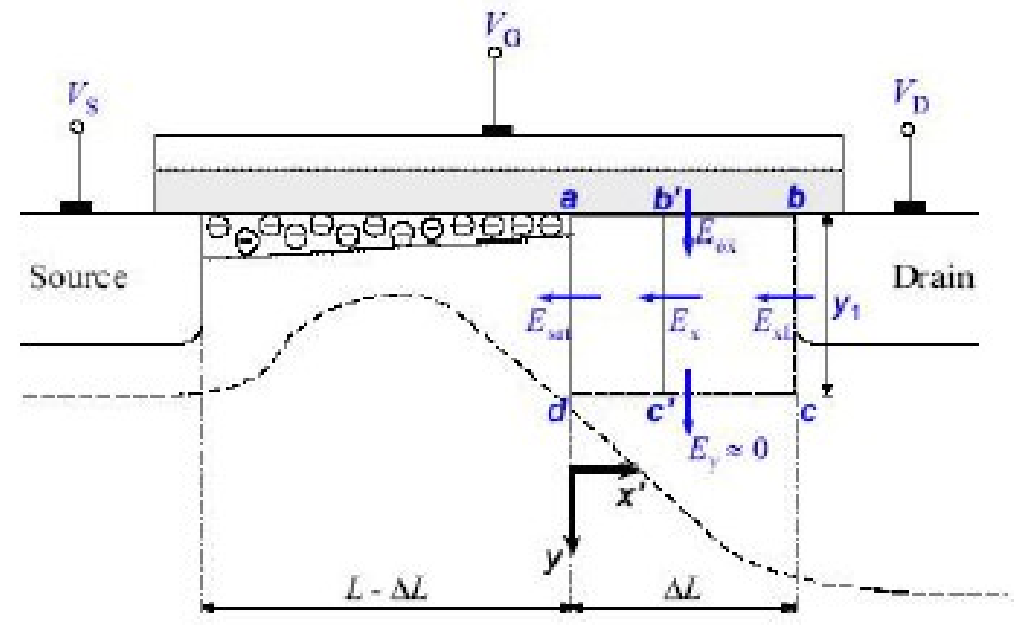

Figure 4 : Illustration de la distance de modulation $\Delta L$

$y_{l}$ est la profondeur avec laquelle le champs électrique $E_{y}$ s'annule. A la frontière a-b désignant la longueur $\Delta \mathrm{L}$ les porteurs de charge atteignent leur vitesse de saturation avec un champ de saturation latéral $E_{\text {sat }}$. Nous considérons que les deux champs électriques sont indépendants.

Le canal est modulé avec une distance $\Delta \mathrm{L}$ donnée par :

$$
\Delta L=\int_{\Psi_{\text {Ssat }}}^{\Psi_{S_{L}}}-\frac{d \Psi_{S}}{E_{x}}
$$

Afin de résoudre cette équation nous devons déterminer la relation entre le potentiel de surface et le champ électrique unidimensionnel suivant l'axe (OX). Appliquons la loi de Gauss à la section a-b'-c'-d.

$$
\int_{0}^{y_{1}}\left(\frac{\partial \Psi_{S}}{\partial x}+E_{s a t}\right) d y+\int_{0}^{x} \frac{\varepsilon_{o x}}{\varepsilon_{S i}} E_{o x} d x=\frac{1}{\varepsilon_{S i}} \int_{0}^{x}\left(q N_{A}^{*} y_{1}-Q_{i n v}\right) d x
$$




$$
\text { avec } \quad E_{o x}=\frac{Q_{g}}{\varepsilon_{o x}} \text {. }
$$

Nous supposons que la variation longitudinale du potentiel de surface ne change pas en fonction de $y$ dans la zone de charge d'espace. Ainsi nous pouvons écrire :

$$
\begin{aligned}
& \int_{0}^{y_{1}} \frac{\partial \Psi_{S}}{\partial x} d y=k y_{1} \frac{\partial \Psi}{\partial x} \\
& k y_{1} \frac{\partial \Psi_{S}}{\partial x}=\int_{0}^{x}-\frac{Q_{g}}{\varepsilon_{S i}} d x+\frac{1}{\varepsilon_{S i}} \int_{0}^{x}\left(q N_{A} y_{1}-Q_{i n v}\right) d x \\
& \frac{\partial^{2} \Psi_{S}}{\partial x^{2}}=-\frac{1}{k \varepsilon_{S i} y_{1}}\left(Q_{g}-q N_{A}+Q_{i n v}\right)
\end{aligned}
$$

Où :

$$
\begin{aligned}
& N_{A}: \text { la concentration des dopants, } \\
& k=I / 3 \text { pour une distribution parabolique, } \\
& \varepsilon_{s i}: \text { Permittivité du silicium, } \\
& \varepsilon_{o x:}: \text { Permittivité de l'oxyde. }
\end{aligned}
$$

Intégrons l'équation (20):

$$
\left.\int_{E_{s a t}^{2}}^{E^{2}} d E_{x}^{2}=-\frac{2}{k y_{1} \varepsilon_{S i}} \int_{\Psi_{s a t}}^{\Psi_{S}} \mid Q_{g}-q N_{A} y_{1}+Q_{i n v}\right) d \Psi_{S}
$$

La modulation de la longueur du canal peut alors être décrite par l'équation suivante :

$$
\frac{\Delta L}{L}=\alpha \ln \left[\frac{V_{D S}-V_{D S_{s a t}}+\sqrt{\left(V_{D S}-V_{D S_{s a t}}\right)^{2}+V_{p}^{2}}}{V_{p}}\right]
$$

où $\alpha$ et $V p$ sont des paramètres propres au modèle utilisé et $\alpha$ inversement proportionnelle à la longueur du canal $L$.

L'équation (22) indique la liaison directe de la modulation de la longueur du canal avec la tension $V_{D S}$.

Les résultats de simulation que nous avons obtenus mettant en évidence la modulation de la longueur du canal en fonction de la tension $V_{D S}$ pour différents dopages sont ceux de la Figure 5. Ces résultats confirment que cette distance augmente avec la tension drain source jusqu'à ce que celle-ci atteigne la tension de saturation qui est de $1.5 \mathrm{~V}$ pour le NMOS et de $-2 \mathrm{~V}$ pour le PMOS. 

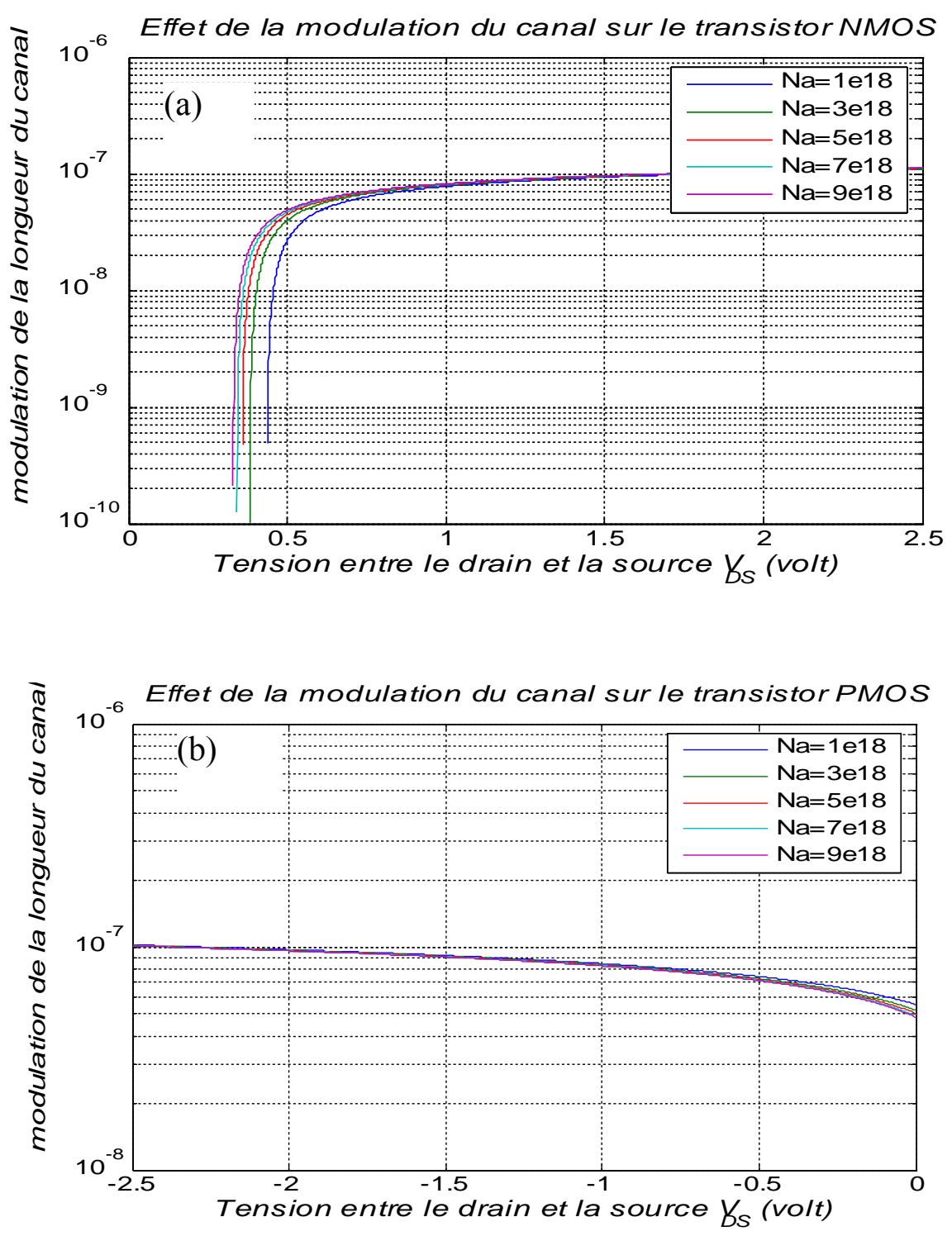

Figure 5 : Effet de la modulation de la longueur du canal pour les transistors NMOS et PMOS

\section{Le bruit thermique (Johnson noise)}

\section{5-1. Introduction au bruit thermique}

En augmentant la fréquence le bruit en $l / f$ devient de plus en plus faible. En radiofréquence ce bruit est négligeable. Le bruit thermique ou bruit de Johnson est dû 
principalement à l'agitation thermique des porteurs de charge. Dans un transistor MOSFET ce bruit est modélisé par la densité spectrale du courant circulant dans le canal du drain vers la source.

\section{5-2. Calcul de la densité spectrale du bruit thermique}

La densité spectrale du bruit thermique est donnée par [6] :

$$
S_{t h_{D}}=\frac{4 k_{B} T}{I_{D S} L^{2}} \int_{V_{S B}}^{V_{D B}} g^{2}(V) d V
$$

où $g(V)$ représente la conductance à chaque point du canal :

$$
g(V)=-\mu(V) W Q_{\text {inv }}(V)
$$

Nous pouvons évaluer cette densité en fonction du potentiel de surface :

$$
S_{t h_{I_{D}}}=\frac{4 k_{B} T}{I_{D S} L^{2}} \int_{\Psi_{S_{0}}}^{\Psi_{S_{L}}} \mu_{e f f}^{2} W^{2} Q_{i n v}^{*} Q_{i n v}-\left(\frac{\mu_{e f f}}{V_{s a t}} I_{D S}\right)^{2} \frac{Q_{i n v}}{Q_{i n v}^{*}} d \Psi_{S}
$$

Ce modèle décrit avec grande précision le bruit thermique pour différentes technologies CMOS. Ceci a été prouvé par des résultats expérimentaux [8].

\section{Effet de la longueur du canal}

Le bruit dans le canal est un bruit blanc, ce bruit peut être inclus dans le circuit équivalent du MOSFET et ceci en ajoutant une source de courant de bruit entre le drain et la source.

\section{6-1. Théorie du MOS à canal long}

La théorie classique des transistors à effet de champ proposée par Van Der Ziel [2] indique que dans de telles structures on trouve deux sources principales du bruit. La première source est représentée par le courant circulant au niveau du drain. Ce bruit est dô à la conductance du canal tandis que la deuxième source est le courant induit de la grille. Ce dernier est le résultat de la fluctuation de la charge dans le canal.

Le courant los est donné sous la forme d'une équation différentielle du premier ordre [2] :

$$
I_{D S}(y)=W Q_{c h_{0}}(y)\left(1-\frac{V_{F(y)}}{V_{b}}\right) \frac{\mu_{e f f}}{1+\frac{E_{y}}{E_{s a t}}} \frac{d V_{F}(y)}{d y}
$$



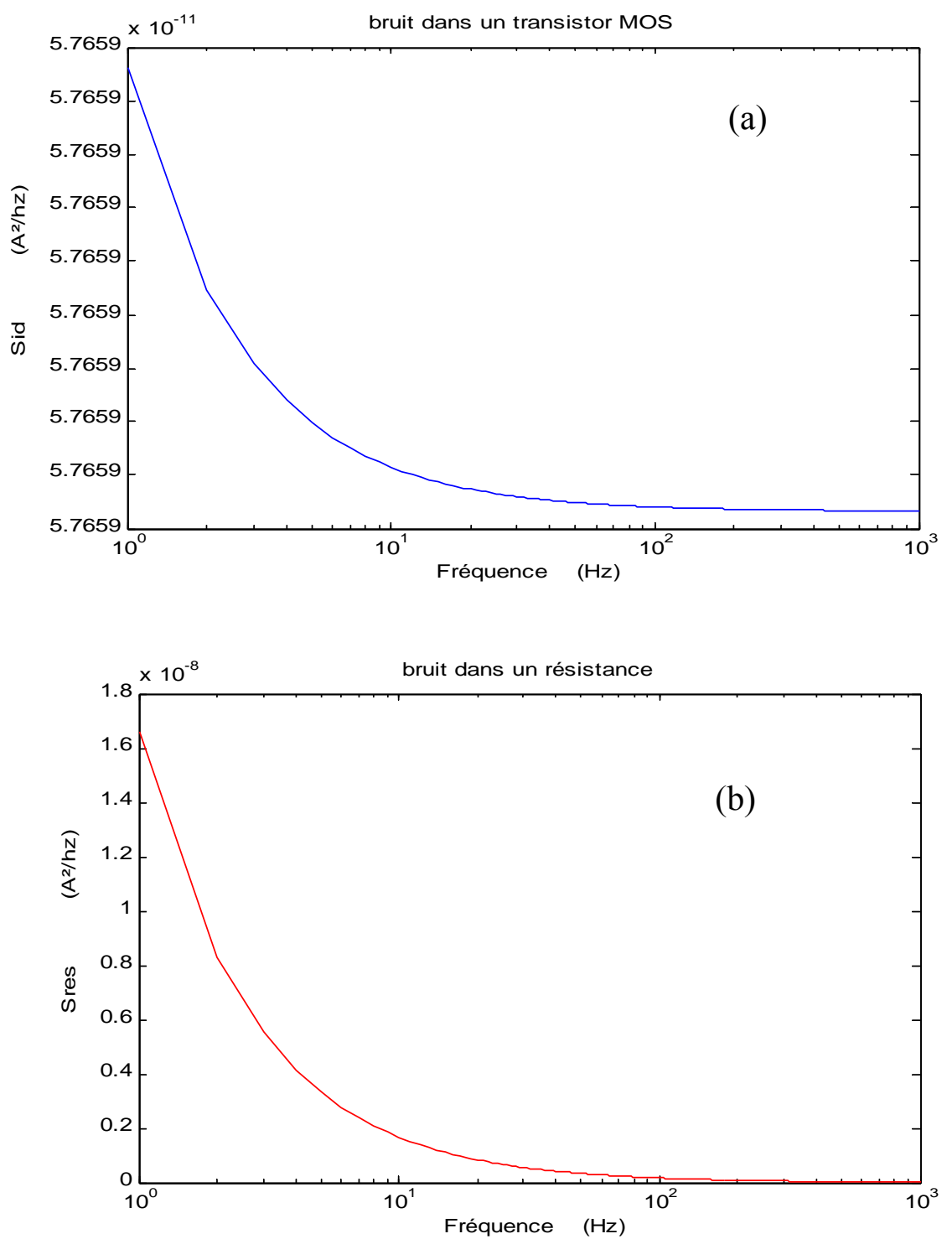

Figure 6 : Comparaison du bruit thermique : (a) dans un transistor MOS (b) dans une résistance

Le canal peut être vu comme une série de résistance où à chacune d'elle on associe une source de bruit thermique telle que :

$$
\overline{v^{2}}=4 k T R \Delta f
$$




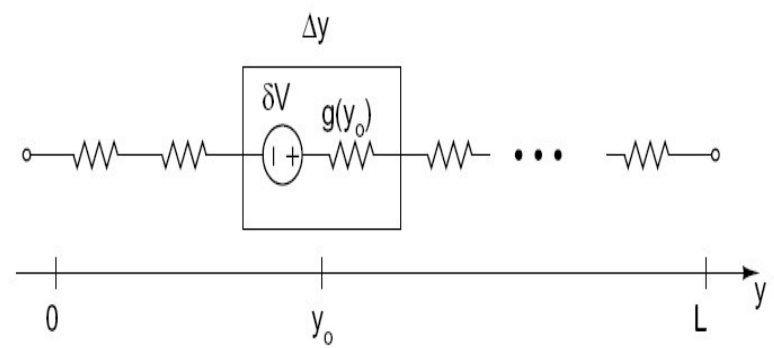

Figure 7 : Présentation du canal comme une série de résistances

Le potentiel dans le canal peut être calculé en chaque point. La distribution du potentiel est donnée par : $\Delta V=V(y)-V_{0}$ où $V_{0}$ représente le potentiel initial.

\section{6-2. Théorie du MOS à canal court}

Le modèle conventionnel du bruit dans le canal des transistors à canal long [9], basé sur la théorie de Nyquist et sur les caractéristiques en mode dc (continu) a prévu avec succès le bruit du canal des transistors à canal court opérant en zone linéaire. Cependant pour les circuits intégrés fonctionnant en radio fréquence, les transistors fonctionnent en zone de saturation pour la plupart des applications. On a pu observer que le bruit du canal produit dans les transistors à canal court, fonctionnant dans la région de saturation, est plus important que le bruit prévu par les modèles conventionnels de bruit du canal. Par conséquent, il devient crucial d'expliquer l'origine de l'augmentation du bruit dans les transistors MOS à canal courts afin de modéliser le bruit du canal des transistors MOSFET submicroniques profonds.

L'approche que nous considérons $[10,12,15]$ consiste à développer un modèle de bruit basé sur un modèle de canal à deux sections, dans lequel le canal du MOSFEl est devisé en 2 régions : région 1 à canal graduel d'une longueur $\mathrm{L}_{\text {elec }}=\mathrm{L}-\Delta \mathrm{L}$ et une région 2 de longueur $\Delta \mathrm{L}$ où les porteurs de charges atteignent leur vitesse de saturation (Figure 4). II y a deux raisons physiques proposées pour expliquer l'augmentation du bruit : l'effet des électrons chauds dans la région 2 [10] et l'effet de la modulation du canal en région $l[11]$.

Notons que la loi d'Ohm n'est pas valide dans la région de saturation ce qui nous empêche d'appliquer la théorie du bruit thermique dans cette région, ce qui rend le bruit de diffusion $[13,15]$ la source principale de bruit dans cette région même si ce courant de diffusion est négligeable [14]. 
Utilisons la théorie générale du bruit de diffusion qui nous permet d'écrire [2] :

$$
\overline{i_{n}^{2}}==\frac{4 q^{2} D n A \Delta f}{\Delta x}
$$

$D$ représente la constante de diffusion, $n$ est la densité des porteurs de charges, $A$ est la surface de la section du canal en question.

Le courant du drain est proportionnel à la charge $Q$, dans le canal, ce courant est donné par :

$$
I_{D}=W Q_{I} v_{\text {sat }}
$$

De cette équation nous pouvons conclure que si les charges dans le canal sont constantes alors le courant du drain est constant et que le courant de la grille est le résultat de la variation de la charge dans le canal.

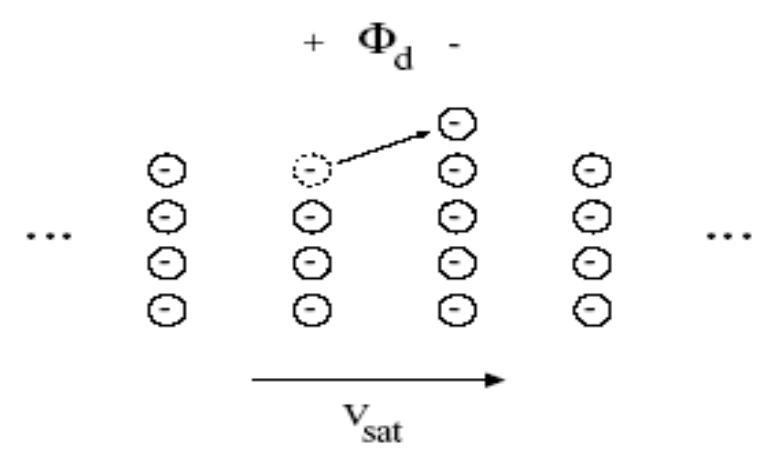

Figure 8 : Présentation du model du bruit

\section{Impact de la résistance du substrat}

\section{7-1. le contexte}

L'étude précédente nous permet de dire que le bruit au niveau du courant du drain d'un transistor MOS à canal court est plus important que celui dans un dispositif à canal long. Ce courant est indépendant de la fréquence. Des résultats expérimentaux [4] montrent que dans la gamme de fréquence $\mathrm{GHz}$ ce courant dépend de la fréquence. Ces résultats ont été considérés comme des erreurs de mesure. Dans ce paragraphe, nous essayons de trouver l'origine physique de la dépendance du courant $I_{D}$ de la fréquence. 


\section{7-2. Modèle du courant du drain}

Considérons la structure simple du transistor MOS donnée par la Figure 9:
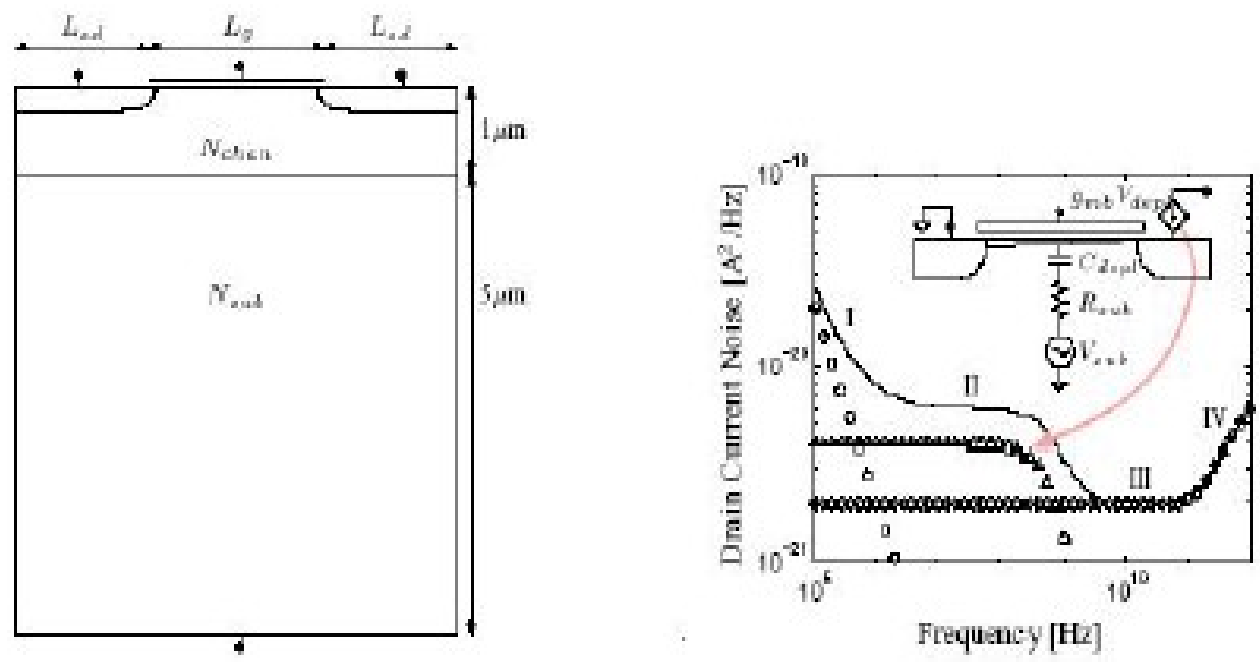

Figure 9 : présentation d'un model simple du transistor MOS

Une telle structure est modélisée par un filtre passe bas et une source de bruit. Le couplage capacitif entre la grille et le canal est l'origine de la propagation des fluctuations et par conséquent de l'augmentation du bruit thermique dans le canal. En plus la résistance du substrat augmente à son tour ce bruit dont l'expression est [3] :

$$
S_{v s u b}=4 k T R_{\text {sub }}
$$

Où $R_{\text {SUB }}$ est la résistance du substrat.

Ces fluctuations modulent les charges de la capacité de déplétion et produit par suite un bruit additif noté $S_{i d, s u b}$ amplifié par la transconductance $g_{m b}$. Ainsi le bruit total dans le canal est donné par :

$$
S_{i s, s u b}=\frac{4 k T R_{\text {sub }} g_{m b}^{2}}{1+\left(w R_{\text {sub }} C_{\text {depl }}\right)^{2}}
$$

\section{Modélisation du bruit induit de la grille}

En haute fréquence le couplage capacitif entre la grille et le canal engendre un bruit dans le courant $I_{G}$. Ce dernier est dit le bruit induit de la grille. 


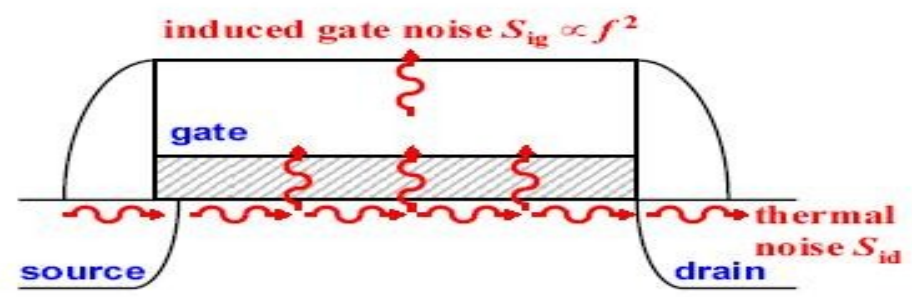

Figure 10 : Représentation du bruit induit de la grille

Le calcul de la densité spectrale du bruit induit de la grille en haute fréquence nous amène à un travail très fastidieux et très difficile pour qu'il soit appliqué dans un circuit de simulation.

Ainsi il est plus pratique de développer un modèle équivalent. Ce dernier est donné par la figure suivante :

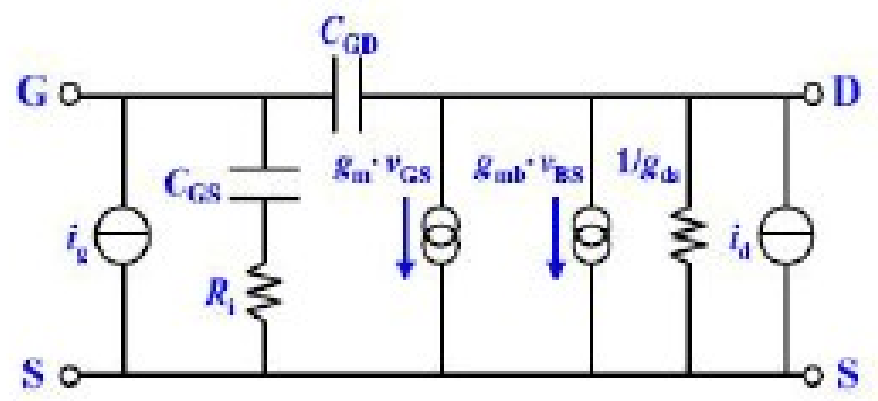

Figure 11 : présentation du modèle équivalent d'un transistor ayant un bruit induit de la grille

En régime de saturation la résistance $R_{\mathrm{i}}$ est donnée par : $R_{i}=\frac{1}{3 g_{m}}$. La densité spectrale du bruit induit de la grille est donnée par la formule suivante :

$$
S_{I_{G}}=4 k_{B} R_{i} w^{2} C_{o x}^{2}
$$




\section{Conclusion}

Dans ce travail nous avons modélisé la densité spectrale des deux sources principales du bruit dans la structure MOS qui sont le bruit en $l / f$ et le bruit thermique pour les différentes régions de fonctionnement. Les résultats sont jugés satisfaisants en regard aux valeurs expérimentales de la littérature. Nous avons démontré que le bruit dans un transistor MOS à canal court est plus important que celui dans un canal long. La résistance du substrat engendre de son coté un bruit dans la structure MOS mais seulement lorsque le dispositif fonctionne dans une gamme de fréquence en $\mathrm{GHz}$. Nous avons aussi noté que le bruit thermique augmente par un couplage capacitif de la grille vers le substrat.

\section{Références}

[1]- Andries J. Scholten, Luuk F. Tiemeijer. "Noise modeling for RF CMOS circuit simulation". IEEE Trans Electron Devices, Vol, 50 No. 3, March 2003

[2] - Todd C. Sepke.."Investigation of noise sources in scaled CMOS Field-Effect Transistors. Master of science in Electrical Engineering and computer sciences", Massachusetts Institute of Technology, June (2002)

[3] - Reza Navid \& Robert W. Dutton. "The physical phenomena responsible for excess noise in short-channel MOS devices". Center for integrated Systems, Stanford University, May (2000)

[4] - Jung-Suk Goo, Simona Donati. "Impact of Substrate Resistance on Drain current Noise in MOSFET's". Center for integrated Systems, Stanford University, Proceeding of SISPAD (2001)

[5] - R. Van Langevelde, A. J. Scholten and D. B .M. KLasseen "MOS model 1100 manual'. June (2004)

[6] - R. Van Langevelde, A. J. Scholten and D. B .M. KLasseen. "Physical background of MOS model II", April (2003)

[7] - [Available]: http://www.semiconductors.philips.com/philips_models

[8] - A. Scholten. "Accurate thermal noise Model for Deep-Submicron CMOS". IEDM technologies, (1999)

[9] - Y. Tsividis," Operation and Modeling of the MOS Transistor", 2" ed, M. J. Deen qnd T.Fjeldym, Eds. New York, Mc Graw-Hill (1999)

[10] - W. Jin, P. C. H. Chan, and J; Lau, "A physical thermal noise model for SOI MOSFET" IEEE Trans. Electron devices, Vol.47, $\mathrm{N}^{\circ} 4$ (April 2000), pp. 768-773 
[11] - G. Knoblinger, P. Klein, and M. Tiebout, "A new model for thermal channel noise of deep submicron MOSFETs and it's application in RF- CMOS design" IEEE J. Solid State Circuits, Vol. $36, \mathrm{~N}^{\circ} 5$ (May 2001), pp. 831-837

[12] - K. Han, H. Shin and K. Lee, "Analytical drain thermal noise current model valid for deep submicron MOSFET" IEEE Trans. Electron devices, Vol.51, $\mathrm{N}^{\circ} 2$ (Feb 2004), pp. 261-269

[13] - E. Statz, H. A. Hoaus and R. A. Pucel, " Noise characteristics of Gallium Arsenide Field Effect transistors", IEEE Trans. Electron devices, Vol. Ed 21, N 9 (Sept. 1974), pp. 549-562

[14] - A. Van der Ziel and K. M Van Vliel " H, F thermal Noise in space charge limited solid state diodes- II", Solid State Electron, Vol.50, $\mathrm{N}^{\circ} \mathrm{l}$ (1968), pp. 508-509

[15] - Y. Cheng, M. J. Deen and C.H Chen, "MOSFET modeling for RF IC design" IEEE Trans. Electron devices, Vol.52, $\mathrm{N}^{\circ} 7$ (July 2005), pp. 1286-1302 